www.jmscr.igmpublication.org

Impact Factor (SJIF): 6.379

Index Copernicus Value: 79.54

ISSN (e)-2347-176x ISSN (p) 2455-0450

crossrefDOI: https://dx.doi.org/10.18535/jmscr/v6i11.18

Journal Of Medical Science And Clinical Research

\title{
Renal Malakoplakia- A Case Report
}

\author{
Authors \\ Dr M.M.Litake ${ }^{1}$, Dr Ashutosh Kumar ${ }^{2}$
}

\begin{abstract}
Malakoplakia is a rare granulomatous disease of infectious etiology. The name is derived from the Greek malakos (soft) and plakos (plaque), describing its usual clinical presentation as friable yellow soft plaques. It was first described by Von Hansemann in 1901 and in 1902 by Michaelis and Gutmann. Total number of patients with malakoplakia is fewer than 500. Most patients have genitourinary tract disease.

We report a case of renal malakoplakia with end stage renal disease in a 42 years old female who presented with pain in the right lower abdomen for about 1 month. Investigations revealed a right kidney in the pelvic region which was non functional on DTPA scan. Right sided open nephrectomy was done. Histopathology suggested malakoplakia with end stage renal disease.

This rare entity is associated with a non- specific clinical presentation. In the imaging studies the appearance of the affected kidneys ranges from a normal kidney to an enlarged, nonfunctioning kidney.
\end{abstract}

\section{Introduction}

The total number of patients with malakoplakia is fewer than 500, including gastrointestinal and skin but most patients of malakoplakia have genitourinary tract disease. The age of diagnosis varies from 6 to 85, with the average age of 50 yrs at presentation.

There is a female preponderance with female to male ratio 4:1. Malakoplakia is believed to result from the inadequate killing of bacteria by macrophages or monocytes that exhibit defective phagolysosomal activity.

Malakoplakia is associated with urinary tract infections in majority of the cases. Renal failure is a common at the presentation with variable severity. About $40 \%$ patients have some form of immunosuppression, including solid organ transplant. Gram negative bacteria are often associated with malakoplakia. E. coli contributes two third of cases.
Differential diagnoses in radiologic studies include local abscess, granuloma, xanthogranulomatous pyelonephritis, lymphoma and multifocal primary or metastatic tumor.

The distinctive basophilic inclusions with surrounding clear halos known as MichaelisGutmann bodies are found within both the histiocytes and the extra- cellular lay in the stromatolites. A nephrectomy can be a choice for unifocal malakoplakia, but the preoperative diagnosis in appropriate clinical settings can prevent unnecessary surgery.

\section{Case Presentation}

A 42 yrs old female patient presented with right lower abdomen pain for 2 month which was dull aching in nature, non radiating, not associated with meals, partially relieved on medication. No history of burning micturition was present. There was no history of fever or vomitting. There was no 
history of altered bowel habits. She had no significant past medical history. Patient had undergone tubectomy 10 years back. Physical examination revealed her vitals were stable. Her abdomen was not distended, no tenderness was present. Contrast CT scan was suggestive of poorly excreting right pelvic ectopic kidney with features of xanthogranulomatous pyelonephritis/ ?chronic emphysematous pyelonephritis. Urine routine microscopy suggestive of abundant pus cells. Urine culture sensitivity suggestive of insignificant bactiuria.

Histopathalogical examination suggest the external surface is irregular and yellowish. On cut section corticomedullary differentiation is not possible. Also small cysts identified measuring 0.8 and $0.5 \mathrm{~cm}$ in diameter. On microscopic examination many cells show small basophilic round structure (Michaelis Gutmann bodies) in their cytoplasm as well as outside. Suggestive of malakoplakia of kidney with end stage renal disease.
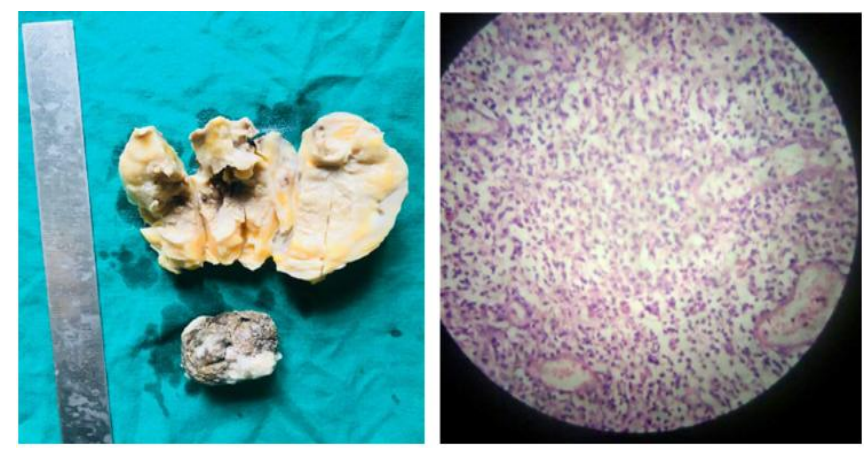

Gross Specimen and Microscopy of Kidney

\section{Discussion}

The patient in our case had a history of dull aching right flank pain for 2 months which could lead to a lot of differential diagnosis like renal calculus, renal tumour, xanthogranulomatous pyelonephritis. The resected mass was grossly similar to a renal mass and the and the yellowish tan colour of the lesion was more like a renal cell carcinoma. On cut section coticomedullary differentiation is not possible and on cut section stone of size $4 \mathrm{~cm}$ noted. Also some small cysts identified measuring $0.8 \mathrm{~cm}$ and $0.5 \mathrm{~cm}$ noted. Pelvis and ureter were not identified. Microscopic examination showed Michaelis Gutmann bodies suggestive of malakoplakia with end stage renal disease.

The treatment of malakoplakia depends on the extent of the disease and the underlying conditions of the patient. Patients with bilateral or multifocal diseases are most often treated with antibiotics such as quinolone. A cholinergic agonist, bethecol chloride, is used to supplement antibiotic regimens to correct the lysosomal defect. Surgical excision is the treatment of choice for unifocal diseases.

\section{Conclusion}

Malakoplakia of the kidney is a rare disease and is diagnosed histopathologically. Other conditions need to be ruled out first.

The large rapidly growing nodules of malakoplakia may mimic renal tumours, especially when ulcerated or accompanied by lymph node involvement.

Pathologists should be alert to the possibility of concomitant renal cell carcinoma. In case of malakoplakia the function of the kidney needs to be assessed as it may lead to end stage renal disease, as in this case and the treatment includes nephrectomy.

\section{References}

1. Wielenberg AJ, Demos TC, Rangachari B, Turk T: Malakoplakia presenting as a solitary renal mass. Am J Roentgenol 2004, 183(6): 1703-1705.

2. Abolhasani et al.: Renal malakoplakia presenting as a renal mass in a 55 year old man: a case report. Journal of Medical Case Reports 2012 6:379

3. Saleem MA, Milford DV, Raafat $F$ et al. Renal parenchymal malakoplakia- a case report and review of literature. Pediatr. Nephrol. 1993: 7: 256-8.

4. Pusl T, Weiss M, Hartmann $B$ et al. Malakoplakia in a renal transplant recipient. Eur. J. Intern .Med . 2006; 17: 133-5. 